\title{
Coronagraphic phase diversity: a simple focal plane sensor for high-contrast imaging
}

\author{
J.-F. Sauvage, ${ }^{1,2, *}$ L. Mugnier, ${ }^{1,2}$ B. Paul, ${ }^{1,2,3}$ and R. Villecroze ${ }^{1,2}$ \\ ${ }^{1}$ Département Optique Théorique et Appliquée, Office national d'études et de recherches aérospatiales (ONERA), \\ Châtillon BP72 92322, France \\ ${ }^{2}$ Groupement d'intérêt scientifique PHASE (Partenariat Haute résolution angulaire Sol Espace) \\ between Office national d'études et de recherches aérospatiales (ONERA), \\ Observatoire de Paris, CNRS, and université Diderot, France \\ ${ }^{3}$ Laboratoire d'Astrophysique de Marseille (UMR7326-CNRS-Institut national des sciences de l'Univers (INSU), \\ Université d'Aix-Marseille), Pôle de l'Étoile Site de Château-Gombert, 38, rue Frédéric Joliot-Curie 13388 Marseille cedex 13, France \\ ${ }^{*}$ Corresponding author: jean-francois.sauvage@onera.fr
}

Received June 28, 2012; revised September 28, 2012; accepted October 2, 2012; posted October 17, 2012 (Doc. ID 171628); published November 16, 2012

\begin{abstract}
Exoplanet direct imaging is a challenging goal of today's astronomical instrumentation. Several high-contrast imaging instruments dedicated to this task are currently being integrated; they are ultimately limited by the presence of quasi-static speckles in the imaging focal plane. These speckles originate in residual quasi-static optical aberrations, which must be measured and compensated for, typically at a nanometric level. We present a novel focal plane wavefront sensor (WFS) designed for this particular application. It is an extension of the phase diversity technique to coronagraphic imaging. This sensor requires no dedicated hardware and uses only two scientific images differing from a known aberration, which can be conveniently introduced by the adaptive optics subsystem. The aberrations are therefore calibrated all the way down to the scientific camera, without any differential aberrations between the sensor and the scientific camera. We show the potential of this WFS by means of simulations, and we perform a preliminary experimental validation. (C) 2012 Optical Society of America

OCIS codes: $\quad 010.7350,100.5070,110.1080,100.3190,350.1260$.
\end{abstract}

Exoplanet direct imaging is a central topic of today's astronomy. The direct imaging of such faint objects, close to their very bright star, requires instruments dedicated to high-contrast imaging [1]. The final detectivity of such a system is determined by the remaining light speckles in the scientific focal plane. These speckles are due to residual optical aberrations in the system. The measurement of aberrations in a high-contrast system is therefore mandatory in order to reach the system final performance. Phase diversity is a focal plane wavefront sensor (WFS) widely used for classical imaging and for multiaperture systems. However, its implementation is currently limited to convolutive cases, for which the point spread function (PSF) is translation invariant. The aim of this Letter is to propose and validate by means of simulations an extension of phase diversity to coronagraphic imaging, hereafter called coronagraphic focal plane wavefront estimator for exoplanet imaging (COFFEE). This WFS will enable the simultaneous characterization of all relevant aberrations with a very simple setup consisting of the existing scientific camera and the existing deformable mirror (DM) of the adaptive optics (AO) subsystem, without removing or modifying any part of the optical setup contrarily to self-coherent camera [2], without any linear approximation as in electric field conjugation [3] and, ultimately, during the scientific acquisitions.

The considered optical coronagraphic imaging system is composed of an AO-capable telescope, a coronagraph, and a detector plane. We distinguish between the upstream aberrations $\varphi_{\text {up }}$ introduced upstream of the focal plane mask and the downstream ones $\varphi_{\text {down }}$. The optical aberrations upstream of the coronagraph are well known to be held responsible for the intensity residuals in the final focal plane image. The measurement and compensation of these aberrations are therefore key points of high-contrast imaging. In this Letter, we assume that all aberrations are quasi-static, i.e., there is no residual turbulence. This description corresponds to a calibration of the aberrations between scientific observations from the ground or to space-based observations.

As in conventional (noncoronagraphic) phase diversity, we shall assume that we use the focal plane camera to record at least two images that differ only from a known aberration, or diversity phase $\varphi_{\text {div }}$, which can be introduced conveniently upstream of the coronagraph by the DM of the AO subsystem. We shall denote by $\boldsymbol{i}_{f}^{c}$ and $\boldsymbol{i}_{d}^{c}$ these focal and phase-diverse coronagraphic images. All aberrations, denoted by $\varphi_{x}$ where $x=$ "up" or "down" or "div," are expanded on a basis $b_{x k}^{k}$, which are typically either Zernike polynomials or the pixel indicator functions in the corresponding pupil plane, $\varphi_{x}(u, v)=$ $\sum_{k} \phi_{x}^{k} b_{x}^{k}(u, v)$, where the summation is, in practice, limited to the number of coefficients considered sufficient to correctly describe the aberrations. We shall denote by $\phi_{x}$ the vector concatenating the set of unknown aberration coefficients $\boldsymbol{\phi}_{x}^{k}$.

Assuming hereafter that the observed object is a point source, a discrete model of these images is

$$
\begin{gathered}
\boldsymbol{i}_{f}^{c}=f \cdot \boldsymbol{h}_{d} \star \boldsymbol{h}_{c}\left(\boldsymbol{\phi}_{\mathrm{up}}, \boldsymbol{\phi}_{\mathrm{down}}\right)+\boldsymbol{n}, \\
\boldsymbol{i}_{d}^{c}=f \cdot \boldsymbol{h}_{d} \star \boldsymbol{h}_{c}\left(\boldsymbol{\phi}_{\mathrm{up}}+\boldsymbol{\phi}_{\mathrm{div}}, \boldsymbol{\phi}_{\mathrm{down}}\right)+\boldsymbol{n}^{\prime},
\end{gathered}
$$

where $f$ is the flux of the source (without coronagraph), $\boldsymbol{h}_{d}$ is the known detector PSF, the star denotes a discrete convolution, and $\boldsymbol{n}$ and $\boldsymbol{n}^{\prime}$ represent the measurement noises. The convolution applied here stands for the action of the pixel response of the detector. 
$\boldsymbol{h}_{c}\left(\boldsymbol{\phi}_{\text {up }}, \boldsymbol{\phi}_{\text {down }}\right)$ is our model for the coronagraphic point source image, also known as "coronagraphic PSF," although it is of course field dependent. In this Letter it is a perfect coronagraph model taken from [ $\underline{4}]$ and depends on $\phi_{\text {up }}$ and $\phi_{\text {down. }}$. Note that this model could also be used for long-exposure images, in which case it has an additional parameter, namely the residual phase structure function.

The measurement noise $\boldsymbol{n}$ (and similarly $\boldsymbol{n}^{\prime}$ ) comprises both photon and detector noises. Because calibration is performed with high photon levels, we adopt a nonstationary white Gaussian model, which is a good approximation of a mix of photon and detector noises. Its variance is the sum of the photon and detector noise variances: $\sigma_{n}^{2}(k, l)=\sigma_{\mathrm{ph}}^{2}(k, l)+\sigma_{\mathrm{det}}^{2}$. The former can be estimated as the image itself thresholded to positive values, and the latter can be calibrated prior to the observations.

We adopt a maximum a posteriori (MAP) approach and estimate the aberrations and the flux $f$ that are most likely given our recorded images and our prior information on the aberrations. This approach boils down to minimizing the neg-log-likelihood of the data, potentially penalized by regularization terms on $\phi_{\text {up }}$ and $\phi_{\text {down }}$ designed to enforce smoothness of the sought phases:

$$
\left(\hat{f}, \hat{\boldsymbol{\phi}}_{\mathrm{up}}, \hat{\boldsymbol{\phi}}_{\mathrm{down}}\right)=\arg \min _{f, \boldsymbol{\phi}_{\mathrm{up}}, \boldsymbol{\phi}_{\mathrm{down}}} J\left(f, \boldsymbol{\phi}_{\mathrm{up}}, \boldsymbol{\phi}_{\mathrm{down}}\right),
$$

where

$$
\begin{aligned}
J\left(f, \boldsymbol{\phi}_{\mathrm{up}}, \boldsymbol{\phi}_{\mathrm{down}}\right)= & \frac{1}{2}\left\|\frac{\boldsymbol{i}_{f}^{c}-f \cdot \boldsymbol{h}_{d} \star \boldsymbol{h}_{c}\left(\boldsymbol{\phi}_{\mathrm{up}}, \boldsymbol{\phi}_{\mathrm{down}}\right)}{\boldsymbol{\sigma}_{n}}\right\|^{2} \\
& +\frac{1}{2}\left\|\frac{\boldsymbol{i}_{d}^{c}-f \cdot \boldsymbol{h}_{d} \star \boldsymbol{h}_{c}\left(\boldsymbol{\phi}_{\mathrm{up}}+\boldsymbol{\phi}_{\mathrm{div}}, \boldsymbol{\phi}_{\mathrm{down}}\right)}{\boldsymbol{\sigma}_{n^{\prime}}}\right\|^{2} \\
& +\mathcal{R}\left(\boldsymbol{\phi}_{\mathrm{up}}\right)+\mathcal{R}\left(\boldsymbol{\phi}_{\mathrm{down}}\right),
\end{aligned}
$$

where $\|x\|^{2}$ denotes the sum of squared pixel values of $\operatorname{map} \boldsymbol{x}, \boldsymbol{\sigma}_{n}$ and $\boldsymbol{\sigma}_{n^{\prime}}$ are the noise variance maps of each image, and $\mathcal{R}$ is a regularization metric for the phase.

In practice, when the phase is expanded on a truncated Zernike basis, the smoothness of the phase is ensured through the basis, and no regularization metric is needed. Conversely, when one wishes to model and reconstruct phases with a high spatial frequency content, the basis of the pixel indicator functions is more appropriate, and one is then led to use a regularization metric such as the one proposed specifically for this basis in Section 8 of [5].

The minimization of metric $J\left(f, \phi_{\text {up }}, \phi_{\text {down }}\right)$ of Eq. (4) is performed by means of a Levenberg-Marquardt algorithm, which is a fast quasi-Newton-type minimization method.

Because this metric is quadratic in $f$, the optimal flux estimate for any current value of $\left(\boldsymbol{\phi}_{\mathrm{up}}, \boldsymbol{\phi}_{\text {down }}\right)$, denoted hereafter by $\hat{f}\left(\boldsymbol{\phi}_{\text {up }}, \boldsymbol{\phi}_{\text {down }}\right)$, can be obtained analytically so that one can perform the minimization on the reduced, somewhat simplified, following metric:

$$
J^{\prime}\left(\boldsymbol{\phi}_{\text {up }}, \boldsymbol{\phi}_{\text {down }}\right)=J\left(\hat{f}\left(\boldsymbol{\phi}_{\text {up }}, \boldsymbol{\phi}_{\text {down }}\right), \boldsymbol{\phi}_{\text {up }}, \boldsymbol{\phi}_{\text {down }}\right) .
$$

The choice of the diversity phase is crucial as it has at least three impacts. Indeed, depending on the chosen diversity, sign ambiguities on the sought phase can be removed or not; second, local minima in the criterion of Eq. (4) can be removed, or at least pushed away from the global minimum, or not; finally, the noise propagation from the images to the estimated phase can vary.

The first item is addressed by choosing two images differing by a known and centrosymmetric phase of diversity, in the same way as in classical phase diversity.

In order to address the second item, we choose a diversity phase such that the criterion is convex on the largest area. An empirical study showed that a mixed diversity composed of astigmatism and focus in the same proportion is appropriate: $\phi_{\text {div }}=0.8 Z_{4}+0.8 Z_{5}$, with $Z_{4}$ and $Z_{5}$ being the Zernike polynomials of focus and astigmatism, respectively. Figure 1 shows a two-dimensional (2D) cut of the criterion with respect to two coefficients of the upstream phase $\phi_{\text {up }}$. For clarity of illustration, a simple case is studied here: the true upstream aberrations are decomposed on the Zernike basis $\phi_{u}=\sum_{k} a_{k} Z_{k}$, and the criterion $J$ is computed in a 2D case, where only two Zernike coefficients $\hat{a}_{4}$ and $\hat{a}_{5}$ are estimated, focus and astigmatism. The upstream estimated aberrations are therefore assumed to be written $\hat{\phi}_{u}=\hat{a}_{4} Z_{4}+\hat{a}_{5} Z_{5}$. The criterion has been computed for values of $a_{4}$ and $a_{5}$ ranging from -2 rad to $2 \mathrm{rad}$ around true values. The upstream aberration is composed of 15 Zernike coefficients, with an RMS value of $0.1 \mathrm{rad}$. The criterion is shown in two cases. In the first one (left) the diversity phase is composed of a sole defocus (as in classical phase diversity), of amplitude $0.8 \mathrm{rad}$. In the second case (right) the diversity phase is composed of $0.8 Z_{4}+0.8 Z_{5}$. Within this range, the criterion shows a global minimum on the expected value for both cases. However, in the left case, it shows several local minima, making the minimization difficult. In the right case, the mixed diversity phase enlarges the global minimum valley and removes some local minima. Indeed, we see that criterion $J\left(f, a_{4} Z_{4}+a_{5} Z_{5}, \phi_{\text {down }}\right)$ remains convex on a radius of about 1 rad around the true phase.

We now validate the COFFEE sensor by simulations. The simulation conditions are the following: we consider Shannon-sampled images at $\lambda=0.635 \mu \mathrm{m}$ with $35 \mathrm{~nm}$ RMS of upstream aberrations, which corresponds to the Spectro Polarimetric High contrast Exoplanet REsearch (SPHERE) specifications. For the proof of

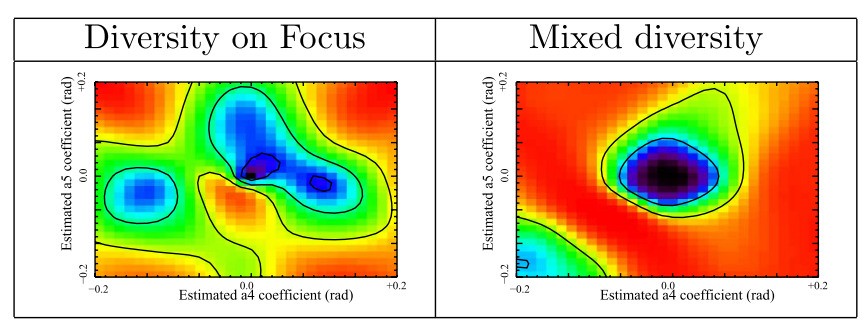

Fig. 1. (Color online) Criterion $J$ to be minimized as a function of the phase parameters $\left(a_{4}, a_{5}\right)$, for an amplitude of the true phase equal to $0.1 \mathrm{rad}$. (Left) The diversity is focus only $\phi_{\text {div }}=0.8 Z_{4}$. (Right) The diversity is a mix of defocus and astigmatism with $\phi_{\text {div }}=0.8 Z_{4}+0.8 Z_{5}$. In both maps, $a_{4}$ and $a_{5}$ range from -2 to +2 rad. 

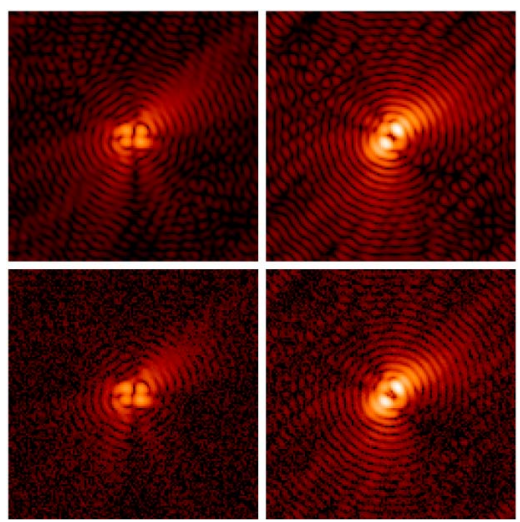

Fig. 2. (Color online) (Bottom) Noisy simulated RRPM focal images and (top) images reconstructed from the estimated aberrations. (Left) Focused and (right) phase-diverse images in logarithmic scale. The simulation accounts for a $2 \times 10^{6}$ photon star, photon and $1 e^{-}$Read-out noise (RON), a perfect RRPM coronagraph, 0.3 rad upstream, 0.1 rad downstream RMS wave-front error (WFE), and a mixed diversity phase.

concept presented herein, these aberrations are limited to 36 Zernike polynomials (up to $Z_{37}$ ). The image simulation includes a fine model of a real coronagraph, namely the Roddier \& Roddier phase mask coronagraph [6] (RRPM). The phase estimation by COFFEE, based on a perfect coronagraph model, will therefore be corrupted by a model error in these simulations. The focal and diverse images are corrupted by photon noise and by an additive white homogeneous Gaussian noise, of standard deviation $\sigma_{d}=1$ electron, corresponding to detector noise. Coronagraphic imaging is known to be notably less sensitive to downstream than to upstream aberrations. Yet we noticed that, although downstream aberrations have no impact on the extinction by the coronagraph, they have a noticeable impact on the detailed shape the images and therefore on aberration estimation. This prompted us to estimate both upstream and downstream aberrations, i.e., to minimize criterion Eq. (5) as a function of both $\phi_{\text {up }}$ and $\phi_{\text {down }}$. It is to be noted that the upstream and downstream aberration include tip and tilt. Upstream tip-tilt characterizes the imperfect centering of the star onto the coronagraph; it is thus of primary importance to be able to measure it. Downstream tip-tilt simply codes for the position of the image on the detector.

The bottom part of Fig. 2 shows coronagraphic images simulated with an RRPM. The upstream and downstream aberrations estimated from these two images, $\left(\hat{\boldsymbol{\phi}}_{\text {up }}, \hat{\boldsymbol{\phi}}_{\text {down }}\right)$, are used as parameters to simulate the two top images of Fig. 2 . A very good match between the RRPM and the reconstructed images can clearly be seen. The reconstruction error is $2.8 \times 10^{-2} \mathrm{rad}$ RMS for the upstream coefficients, which is $4.6 \times 10^{-3} \mathrm{rad}$ per estimated mode, assuming a white repartition of Zernike coefficient on the estimated phase, or a subnanometric error per mode at a wavelength of $635 \mathrm{~nm}$.
Table 1. Introduced Tilt Values and Their Estimation by COFFEE from a Pair of Experimental Images

\begin{tabular}{llllll}
\hline Introduced & -0.839 & -0.666 & -0.488 & -0.317 & $-0.147 \ldots$ \\
Estimated & -0.843 & -0.617 & -0.480 & -0.331 & $-0.176 \ldots$ \\
+0.042 & +0.208 & +0.388 & +0.566 & +0.754 & +0.936 \\
+0.042 & +0.242 & +0.419 & +0.610 & +0.763 & +0.937 \\
\hline
\end{tabular}

The error on the downstream coefficients is $2.9 \times$ $10^{-2}$ rad RMS.

A lab experiment is done to perform a first application of COFFEE to low order estimation, such as tip-tilt. The experimental setup consists in a classical AO bench $(5 \times 5$ subaperture Shack-Hartmann WFS, $6 \times 6$ DM) An apodized RRPM, adapted to a $635 \mathrm{~nm}$ monochromatic light, is placed in the imaging path, downstream of the $\mathrm{AO}$ loop and upstream of the imaging camera. A Lyot stop of $95 \%$ is used. A well-calibrated tilt is introduced upstream of the coronagraphic mask by modifying the mean reference slopes of the closed AO loop. 11 pairs of coronagraphic images are then recorded for different amounts of tilt, ranging from -1 to $+1 \mathrm{rad}$ RMS. From each image pair, 36 Zernike modes are estimated both for the upstream phase and for the downstream aberrations, from tip-tilt to $Z_{37}$. Table 1 shows the introduced and estimated tilt values, given in Z Zernike coefficient ( $\mathrm{rad}$ RMS). The estimation error is evaluated at $0.024 \mathrm{rad}$ RMS on the whole range, which is 0.016 diffraction unit.

We have presented and validated by simulations the principle of a novel WFS for high-contrast imaging called coronagraphic phase diversity, which is an extension of phase diversity to coronagraphic imaging. To our knowledge, a very first experimental validation has also been performed. Although the results presented herein are preliminary, they suggest that coronagraphic phase diversity could be an extremely simple and yet powerful WFS for high-contrast imaging. Perspectives of this work include a complete characterization of COFFEE (aliasing impact, sensitivity to realistic coronagraph, impact of amplitude aberrations, effect of wide band light). Moreover, a more thorough experimental validation with the estimation of higher-order aberrations is mandatory to evaluate the performance of COFFEE.

\section{References}

1. J.-L. Beuzit, M. Feldt, K. Dohlen, D. Mouillet, P. Puget, and F. Wildi, Proc. SPIE 7014, 1 (2008).

2. M. Mas, P. Baudoz, G. Rousset, R. Galicher, and J. Baudrand, Proc. SPIE 7735, 773566 (2010).

3. A. Give'on, R. Belikov, S. Shaklan, and J. Kasdin, Opt. Express 15, 12338 (2007).

4. J.-F. Sauvage, L. M. Mugnier, G. Rousset, and T. Fusco, J. Opt. Soc. Am. A 27, A157 (2010).

5. L. M. Mugnier, A. Blanc, and J. Idier, Advances in Imaging and Electron Physics, P. Hawkes, ed. (Elsevier, 2006), Chap. 1, pp. 1-76.

6. F. Roddier and C. Roddier, Pub. Astron. Soc. Pac. 109, 815 (1997). 